Thorax, 1979, 34, 711-712

\title{
Editorial
}

\section{Prevention of infective endocarditis}

Much thought, conference time, and journal space has been devoted to this topic in recent years. The problem not only remains unsolved, it does not appear even to have been dented. The most recent recommendations of the American Heart Association (1977) are unlikely to have helped.

When bacterial endocarditis was first described by Horder (1908-9) and by Osler (1908-9) at the turn of the century the typical victim was a young adult with rheumatic heart disease and a streptococcal endocarditis. The disease was then a fatal one. Whereas rheumatic fever has virtually disappeared from Britain, apparently as a result of penicillin, the incidence of streptococcal endocarditis has barely diminished and still carries a mortality close to the $30 \%$ to which it fell soon after penicillin was introduced in 1944. A priori, mortality figures refer to recognised cases but just as late diagnosis accounts for this continuing high mortality, no diagnosis may be responsible for a true mortality that is even higher. Yet diagnosis need not be so difficult nor treatment too late, and surely prevention too is possible?

In Britain streptococci continue to cause the vast majority of "medical" infections. Except in the geriatric age group these streptococci are largely of the viridans group and are penicillin sensitive. Even in geriatric patients with their higher incidence of enteric organisms Streptococcus viridans still accounts for more than $50 \%$ of cases.

"Surgical" endocarditis is numerically less common and includes both early postoperative cardiac cases and patients who develop endocarditis after a skin incision for any other purpose, even piercing of ears and accidental wounds. Surgical endocarditis is mainly staphylococcal with a particularly high and important incidence of Staphylococcus epidermidis. Cardiac surgery, invariably carried out under broad-spectrum antibiotic "cover," may be complicated by candida or other opportunistic organisms, particularly when the operation is prolonged. Cardiac surgery poses quite different considerations in prophylaxis, as do investigations such as cystoscopy and transrectal biopsy of the prostate gland, which are well-based antecedents of infective endocarditis in elderly men. Even a barium enema can be followed by transient bacteraemia, so where should prophylaxis stop?

Why is $S$ viridans endocarditis still so common? An oral portal of entry has been assumed and dental extraction blamed. Prophylactic penicillin was long ago advocated to cover dental operations in susceptible subjects. We can examine the possible mechanisms for failure:

(1) Perhaps the penicillin is not given. In his sample survey of dentists' practice in this regard Durack (1975) showed that although only a minority of patients received intramuscular penicillin within an hour of extraction as recommended by Garrod and Waterworth (1962), oral penicillin was usually given. This was often started too early and theoretically could have induced an increased incidence of penicillin-resistant infections.

(2) Perhaps dental treatment is not after all to blame. Although streptococcal bacteraemia occurs during dental extraction and its incidence is reduced by penicillin, a similar bacteraemia can also be shown in some people during chewing.

(3) Perhaps the wrong population is being protected. "Susceptible subjects" have in the past been regarded as patients with a known cardiac abnormality, either congenital or acquired. Only last year Petersdorf (1978) suggested that penicillin prophylaxis should be extended to include any one with a murmur or even a history of rheumatic fever. Yet nearly $50 \%$ of cases of infective endocarditis have not previously been known to have heart disease and between 25 and $40 \%$ of cases develop on normal valves (although these are usually leaking by the time the diagnosis is made).

If normal hearts are susceptible to infection and chewing can cause bacteraemia then the only means of reducing the incidence of streptococcal endocarditis that is likely to be effective is to raise the standard of oral hygiene throughout the country and to "cover" all causes of predictably heavy bacteraemia with appropriate antibiotics.

Certainly some cardiac abnormalities seem to be unduly vulnerable (Rodbard, 1963), especially congenitally bicuspid aortic valves; some infections are especially hard to eradicate, such as those on artificial valves; and some individuals appear to be 
especially susceptible and have repeated episodes of endocarditis despite every care. But minor congenital aortic valve anomalies are usually unrecognised, so that their possessors are not selected for prophylaxis, while patients with valvar prostheses usually have their dental treatment in hospital where optimal antibiotic cover should present no difficulty.

What is optimal prophylaxis, and which individuals should be selected to receive it? In 1977 the American Heart Association published its revised recommendations. Despite the logistic nearimpossibility of organising correctly timed parenteral antibiotic prophylaxis to precede dental operations outside hospital the committee proposed intramuscular penicillin followed by eight doses of phenoxymethyl penicillin $0.5 \mathrm{~g}$ six hourly. Patients admitting to penicillin sensitivity were to receive intravenous vancomycin followed by erythromycin. Venturing further into impracticality Kaye (1976) had earlier proposed even larger doses of parenteral antibiotics continued for three days. Fortunately, Shanson et al (1978) have recently shown that a single large oral dose of amoxycillin, $2 \mathrm{~g}$ one hour before extraction, reduced the observed incidence of bacteraemia from 14 out of 14 in control subjects to two out of 14 given amoxycillin. As would be expected phenoxymethyl penicillin was less effective.

It seems most practical to advise a single $2 \mathrm{~g}$ dose of oral amoxycillin before dental extractions or scaling out of hospital, and to retain intramuscular penicillin plus gentamicin for inpatients. Penicillin-sensitive subjects should be given erythromycin. Although previous recommendations have always been for antibiotics to be given only to individuals who had been recognised to be "at risk", such selection was based on false concepts and has not worked. Logic suggests that prophylaxis should be given to everybody. Such a policy would be cheap, easy, and effective.

Investigations and operations on the urinary and lower alimentary tracts are invariably carried out in hospital, where relevant parenteral bactericidal antibiotic prophylaxis should be given to prevent enterococcal infection. This will usually be ampicillin plus gentamicin and again should be given unselectively to all. A single dose suffices to cover investigations and Petersdorf suggested 12 to 24 hours for surgical procedures.

The question of whether antibiotics should be given to cover normal childbirth is more vexed. Although transient bacteraemia may accompany normal childbirth, infective endocarditis is so rare after parturition that not all cardiologists and ob- stetricians feel that prophylaxis is necessary, while on the previous arguments it would not be logical to limit its use to women with known heart disease.

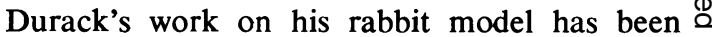
criticised on the grounds that his was an exercise \& in early treatment rather than in prevention of in- $\overrightarrow{0}$ fective endocarditis (Durack, 1975). This brings the argument back full circle because the fore- $\vec{\omega}$ going considerations indicate that prevention may not be wholly feasible, even in the case of strepto- $x$ cocci of reliable penicillin sensitivity, and even less $\omega_{\perp}$ so for the numerically smaller group of unpre- $\sigma$ dictable infections caused by more exotic organ- $\stackrel{\sim}{\exists}$ isms such as coxiella or chlamydia.

Early treatment remains the cornerstone in the $\frac{\text { ? }}{?}$ prevention of death and chronic disability from $\vec{r}$ infective endocarditis. The widespread use of anti- $\mathbb{D}$ biotics for incompletely diagnosed disorders in $\Phi$ general practice probably cures a number of very $\frac{}{0}$ early cases while dangerously masking others. $A \stackrel{\mathbb{D}}{-}$ common problem is the patient with developed $\vec{\theta}$ disease in whom a clinical diagnosis should have 0 been made and acted on but in whom treatment 0 is still too often delayed, with tragic results, pending the results of blood cultures.

CELIA M OAKLEY

Royal Postgraduate Medical School, $\stackrel{\mathbb{Q}}{\circ}$ Hammersmith Hospital, London W12 OHS

\section{References}

American Heart Association (1977). Committee report: Prevention of bacterial endocarditis. Circulation, 56, 139A-143A.

Durack, D T (1975). Current practice in prevention of bacterial endocarditis. British Heart Journal, 37, ®ֶ 478-481.

Garrod, L P, and Waterworth, M M (1962). The risks of dental extraction during penicillin treatment. British Heart Journal, 24, 39-46.

Horder, T (1908-9). Infective endocarditis with an 을 analysis of 150 cases and with special reference to the chronic form of the disease. Quarterly Journal of Medicine, 2, 290-324.

Kaye, D (ed) (1976). Infective Endocarditis. University N Park Press, Baltimore.

Osler, W (1908-9). Chronic infective endocarditis. O Quarterly Journal of Medicine, 2, 219-230.

Petersdorf, R G (1978). Antimicrobial prophylaxis of 0 bacterial endocarditis. Prudent caution or bacterial overkill? A merican Journal of Medicine, 65, 220-234.

Rodbard, S (1963). Blood velocity and endocarditis. Ф Circulation, 27, 18-28.

Shanson, D C, Cannon, P, and Wilks, M (1978). Jour- $\frac{0}{-}$ nal of Antimicrobial Chemotherapy, 4, 431-436.

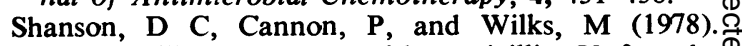
Amoxycillin compared with penicillin $V$ for the $\stackrel{\odot}{\mathscr{Q}}$ prophylaxis of dental bacteraemia. Journal of Antimicrobial Chemotherapy, 4, 431-436. 